\title{
Temperature characteristics of epitaxially grown InAs quantum dot micro-disk lasers on silicon for on-chip light sources
}

\section{Citation}

Wan, Yating, Qiang Li, Alan Y. Liu, Arthur C. Gossard, John E. Bowers, Evelyn L. Hu, and Kei May Lau. 2016. "Temperature Characteristics of Epitaxially Grown InAs Quantum Dot Micro-Disk Lasers on Silicon for on-Chip Light Sources." Applied Physics Letters 109 (1) (July 4): 011104. doi:10.1063/1.4955456.

\section{Published Version}

doi:10.1063/1.4955456

\section{Permanent link}

http://nrs.harvard.edu/urn-3:HUL.InstRepos:33892597

\section{Terms of Use}

This article was downloaded from Harvard University's DASH repository, and is made available under the terms and conditions applicable to Other Posted Material, as set forth at http:// nrs.harvard.edu/urn-3:HUL.InstRepos:dash.current.terms-of-use\#LAA

\section{Share Your Story}

The Harvard community has made this article openly available.

Please share how this access benefits you. Submit a story.

Accessibility 


\title{
Temperature characteristics of epitaxially grown InAs quantum dot micro-disk lasers on silicon for on-chip light sources
}

\author{
Yating Wan, ${ }^{1, a)}$ Qiang Li, ${ }^{1, a)}$ Alan Y. Liu, ${ }^{2, a)}$ Arthur C. Gossard, ${ }^{2,3}$ John E. Bowers, ${ }^{2,3}$ \\ Evelyn L. Hu, ${ }^{4}$ and Kei May Lau ${ }^{1, b)}$ \\ ${ }^{1}$ Department of Electronic and Computer Engineering, Hong Kong University of Science and Technology, \\ Clear Water Bay, Kowloon, Hong Kong \\ ${ }^{2}$ Materials Department, University of California Santa Barbara, Santa Barbara, California 93106, USA \\ ${ }^{3}$ Department of Electrical and Computer Engineering, University of California Santa Barbara, Santa Barbara, \\ California 93106, USA \\ ${ }^{4}$ School of Engineering and Applied Sciences, Harvard University, Cambridge, Massachusetts 02138, USA
}

(Received 19 May 2016; accepted 24 June 2016; published online 8 July 2016)

\begin{abstract}
Temperature characteristics of optically pumped micro-disk lasers (MDLs) incorporating InAs quantum dot active regions are investigated for on-chip light sources. The InAs quantum dot MDLs were grown on V-groove patterned (001) silicon, fully compatible with the prevailing complementary metal oxide-semiconductor technology. By combining the high-quality whispering gallery modes and 3D confinement of injected carriers in quantum dot micro-disk structures, we achieved lasing operation from $10 \mathrm{~K}$ up to room temperature under continuous optical pumping. Temperature dependences of the threshold, lasing wavelength, slope efficiency, and mode linewidth are examined. An excellent characteristic temperature $\mathrm{T}_{\mathrm{o}}$ of $105 \mathrm{~K}$ has been extracted. Published by AIP Publishing. [http://dx.doi.org/10.1063/1.4955456]
\end{abstract}

Whispering gallery mode (WGM) micro-resonators incorporating quantum dot (QD) active regions have aroused great interest recently for on chip light sources, due to their low threshold, high temperature stability of operation, and small form factor. ${ }^{1-4}$ While suspended disks with air-clad waveguides provide the best optical confinement, the larger thermal resistance compared to ring resonators may be a drawback. ${ }^{3}$ Room temperature continuous-wave $(\mathrm{CW})$ operation of InAs quantum dot micro-disk lasers (MDLs) has been demonstrated, but only limited research has been carried out on their temperature characteristics. ${ }^{2,4,5}$ Under $\mathrm{CW}$ excitation, Ide $e t a l .{ }^{4}$ reported MDL on a GaAs substrate with $\mathrm{T}_{\mathrm{o}}$ of 64 and $36 \mathrm{~K}$ in the temperature range of $130-230 \mathrm{~K}$ and above $230 \mathrm{~K}$, respectively. Under pulsed excitation, Yang et al. ${ }^{5}$ demonstrated $\mathrm{T}_{\mathrm{o}}$ of $31 \mathrm{~K}$ above room temperature in MDL on a GaAs substrate. A systematic characterization of lasing behavior as a function of temperature for MDLs integrated on a silicon substrate is yet to be investigated.

Recently, we demonstrated InAs quantum dot microdisk lasers grown on a V-groove patterned (001) Si substrate. ${ }^{6}$ In this work, we systematically characterize the lasing behavior of the MDLs, including threshold, lasing wavelength, slope efficiency, and mode linewidth, as a function of temperature. We have obtained an excellent characteristic temperature $T_{o}$ of $105 \mathrm{~K}$ for the epitaxially grown MDLs on $\mathrm{Si}$, which outperform previously reported InAs quantum dot micro-disk lasers on GaAs substrates. ${ }^{4,5,7}$ These temperature insensitive microdisk lasers with compact size and low thresholds directly grown on (001) silicon can be attractive on-chip light sources to meet the lowpower consumption and athermal performance demands of silicon photonic devices.

\footnotetext{
${ }^{a)}$ Y. Wan, Q. Li, and A. Y. Liu contributed equally to this work.

b)Email: eekmlau@ust.hk. Tel.: (852)23587049. Fax: (852)23581485.
}

We first grew $\mathrm{GaAs} / \mathrm{Si}$ templates consisting of $1 \mu \mathrm{m}$ GaAs thin film on a V-groove patterned Si substrate by metal-organic chemical vapor deposition (MOCVD), using the method detailed in Refs. 8 and 9. On the GaAs-on-Si template, microdisk laser structure was deposited in a molecular beam epitaxy (MBE) system. The MBE epi-layers consist of, from bottom to top, a $1 \mu \mathrm{m} \mathrm{GaAs}$ buffer, a $600 \mathrm{~nm} \mathrm{Al} \mathrm{Al}_{0.7} \mathrm{Ga}_{0.3}$ As post region, and a $500 \mathrm{~nm}$ disk region where five-stacked InAs/InGaAs dots-in-a-well (DWELL) structure is sandwiched by $50 \mathrm{~nm} \mathrm{Al}_{0.4} \mathrm{Ga}_{0.6} \mathrm{As}$ cladding layers. ${ }^{10}$ Fig. 1(a) shows the photoluminescence (PL) emission spectra of the as-grown epitaxial structure, with pump power density varied from 20 to $4000 \mathrm{~W} / \mathrm{cm}^{2}$. Ground state emission $(1.29 \mu \mathrm{m})$ is located within the O-band of fiberoptic communications $(1.26-1.36 \mu \mathrm{m})$. The micro-disk lasers were defined using colloidal lithography, followed by $\mathrm{BCl}_{3} / \mathrm{Ar}$ inductively coupled plasma (ICP) etching, as shown in Fig. 1(b). A dilute suspension of $\mathrm{SiO}_{2}$ spheres in isopropyl alcohol (IPA) was dispersed onto the sample surface using a pipette and then rapidly dried on a hot plate at $110^{\circ} \mathrm{C}$. The commercial $\mathrm{SiO}_{2}$ spheres are $4 \mu \mathrm{m}$ in diameter with $\pm 5 \%$ variation. Using the isolated sphere-masks, ICP etching was performed to reach a depth of $\sim 1.1 \mu \mathrm{m}$ into the epi-structure, stopping at the GaAs buffer layer. The disk membrane was suspended upon the virtual GaAs-on-Si substrate by partially etching the underlying $\mathrm{Al}_{0.7} \mathrm{Ga}_{0.3} \mathrm{As}$ sacrificial layer using dilute hydrofluoric (HF) acid. The lateral undercut was designed to be $\sim 1.6 \mu \mathrm{m}$ to guarantee efficient light confinement within the disk perimeter while maintaining good heat sinking through the underlying pedestals. Schematic illustration of the final device structure is presented in Fig. 1(c). Noteworthy is the unique defect trapping effect provided by growing GaAs on V-groove nano-patterned Si substrates, as indicated by the cross-sectional TEM image near the hetero-interface in Fig. 1(d). It allows us to 
$\mathbf{a}$

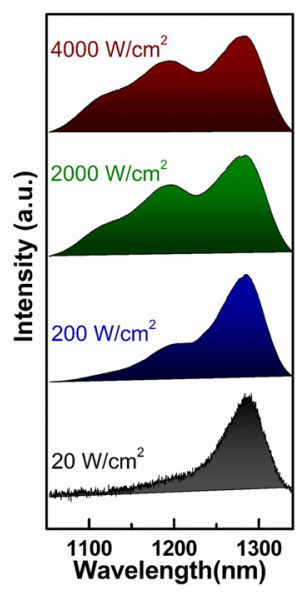

b

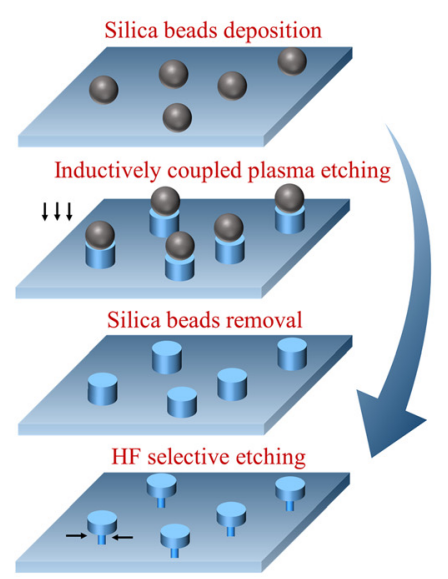

c
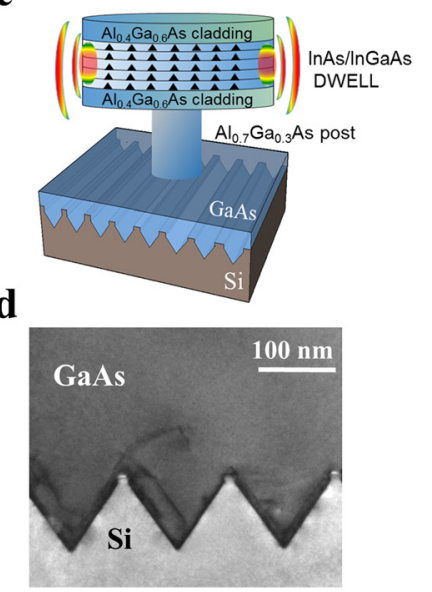

FIG. 1. (a) Room temperature photoluminescence spectra of the as-grown structure at progressively higher excitations; (b) schematic illustrations of the fabrication procedure; (c) schematic illustrations of the fabricated device; and (d) cross-sectional TEM image of the V-grooved structure, showing defect trapping and localization. achieve high quality III-V/Si epitaxy on exact (001) silicon substrates without incorporating intermediate $\mathrm{Ge}$ or other buffer layers. ${ }^{11}$ Better compatibility with the prevailing complementary metal oxide-semiconductor (CMOS) manufacturing processes and silicon-on-insulator (SOI) technologies differentiates this epitaxial growth platform with other quantum dot lasers grown on $\mathrm{Ge}-\mathrm{on}-\mathrm{Si}$ or offcut $\mathrm{Si}$ substrates. ${ }^{12-17}$

The fabricated devices were optically pumped at normal incidence with a CW diode laser operating at $532 \mathrm{~nm}$. The excitation beam was focused to approximately $4 \mu \mathrm{m}$ in diameter. The laser emission was collected through the same objective and analyzed by a monochromator with an InGaAs charge-coupled device array. For temperature dependent measurements, the sample was mounted in a helium gas flow cryostat, with device temperature controlled from $10 \mathrm{~K}$ to room temperature. Fig. 2(a) compares the normalized lasing spectra at three times threshold pump power from 10 to
$300 \mathrm{~K}$. A scanning electron micrograph (SEM) image of an optically isolated, undercut MDL is shown in the inset of Fig. 2(a). The spectra show narrow lines corresponding to high-quality whispering-gallery modes. At $10 \mathrm{~K}$, multimode lasing occurs. The mode at longer wavelengths wins in the mode competition and dominates the spectrum above $80 \mathrm{~K}$. We observe red-shifting of the lasing peak with increasing temperature. Abrupt changes towards longer wavelength cavity modes were also observed due to the effect of the mode overlapping with the gain spectrum. Fig. 2(b) presents the integrated output-power intensity versus the pump power ( $\mathrm{L}-\mathrm{L}$ curve) of the lasing peak from 10 to $300 \mathrm{~K}$. The distinct kinks signify the onset of laser operation over the entire temperature range.

Fig. 3(a) highlights the optical transition positions in the gain medium pumped by the lowest and highest excitation at $10 \mathrm{~K}$. The emission below threshold $(8.25 \mu \mathrm{W})$ was magnified $100 \times$ and plotted together with that well above the

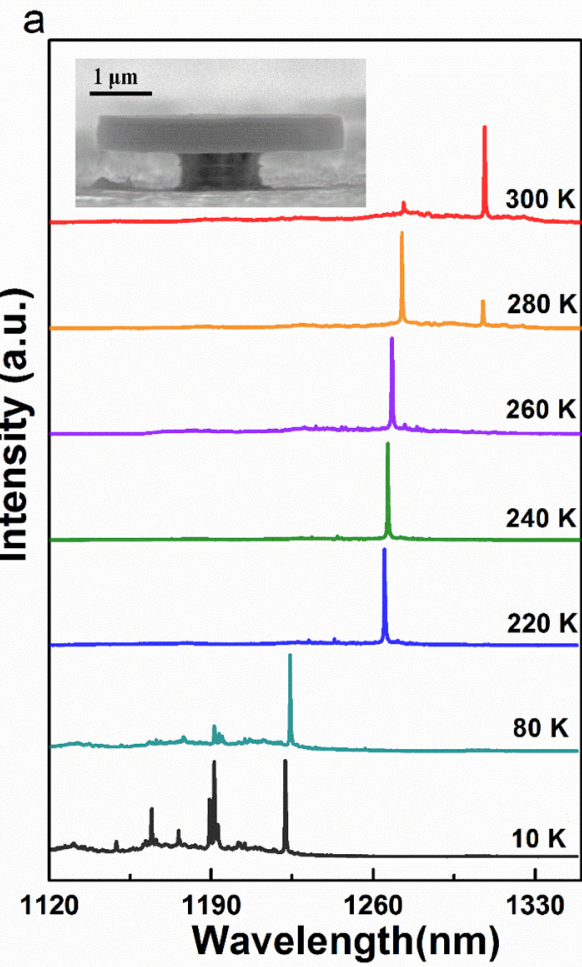

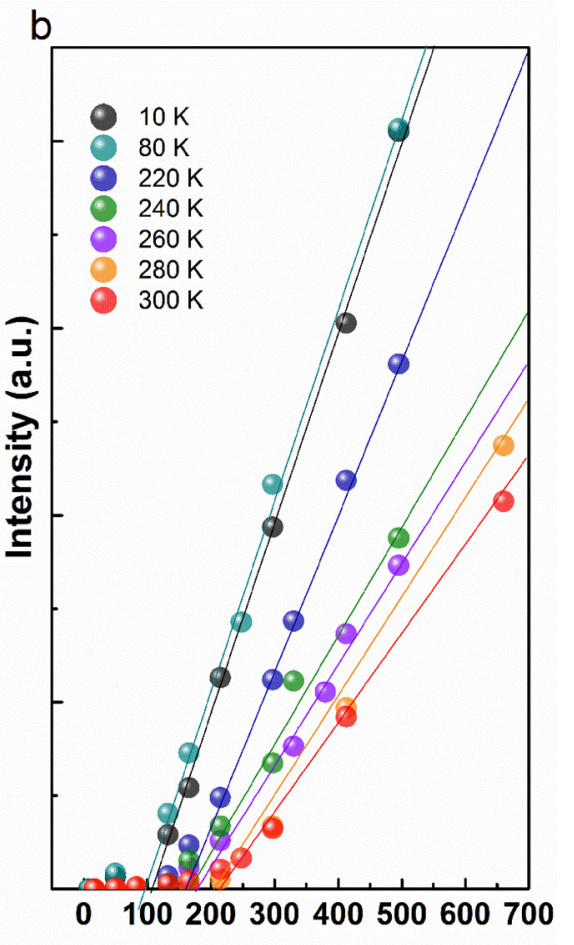

Injection power(uW)
FIG. 2. (a) Normalized lasing spectra at three times the threshold at various temperatures from 10 to $300 \mathrm{~K}$. Inset: $90^{\circ}$ tilted SEM image of the fabricated micro-disk and (b) $\mathrm{L}-\mathrm{L}$ curves of the lasing peak from 10 to $300 \mathrm{~K}$; the solid lines represent linear fits to the experimental data. 
a
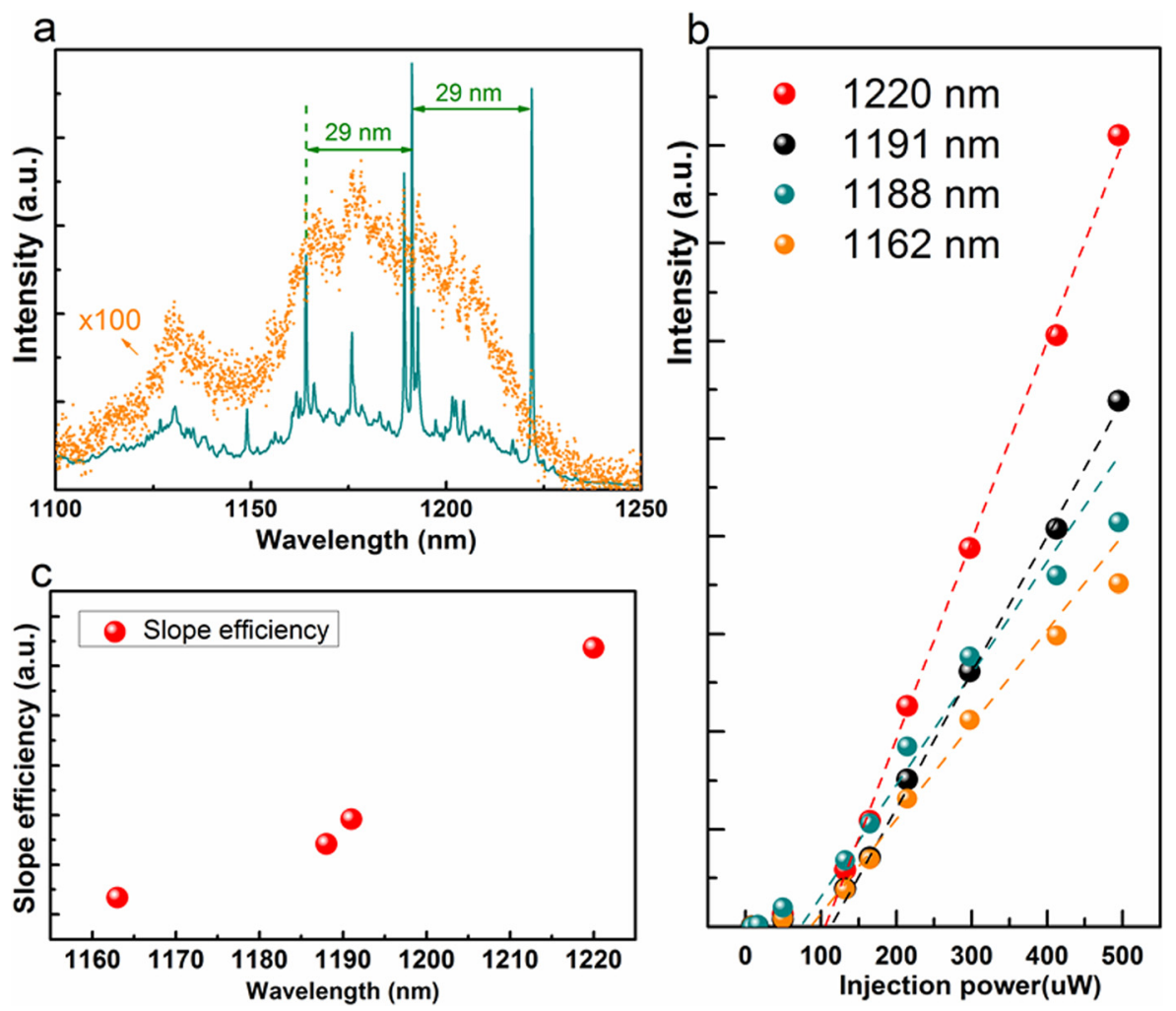

FIG. 3. (a) Laser emission spectra measured below (yellow, $8.25 \mu \mathrm{W}$ ) and above (light blue, $495 \mu \mathrm{W}$ ) threshold in $10 \mathrm{~K}$. Spectrum taken at $8.25 \mu \mathrm{W}$ was amplified 100 times to be visible; (b) $\mathrm{L}-\mathrm{L}$ curves of the lasing peak at $1162 \mathrm{~nm}$, $1188 \mathrm{~nm}, 1191 \mathrm{~nm}$, and $1220 \mathrm{~nm}$ in $10 \mathrm{~K}$, and the dashed lines represent linear fits to the experimental data; and (c) threshold power as well as relative slope efficiency of the four modes at $1162 \mathrm{~nm}$, $1188 \mathrm{~nm}, 1191 \mathrm{~nm}$, and $1220 \mathrm{~nm}$ in $10 \mathrm{~K}$. threshold $(495 \mu \mathrm{W})$. Four lasing peaks at $1162 \mathrm{~nm}, 1188 \mathrm{~nm}$, $1191 \mathrm{~nm}$, and $1220 \mathrm{~nm}$ were observed, evidenced by the pronounced kinks in the L-L curves in Fig. 3(b). The sub-peak $(1188 \mathrm{~nm})$ close to the main peak $(1191 \mathrm{~nm})$ arises from outof-plane polarization. ${ }^{7}$ The other three well-separated modes correspond to WGM with different azimuthal orders, and the mode spacing is extracted to be around $29 \mathrm{~nm}$. Using the equation of free spectral range (FSR) $\Delta \lambda \approx \frac{\lambda^{2}}{2 \pi R n_{e f f}},{ }^{18}$ where R is the radius of the disk and $\lambda$ is the emission wavelength, the effective group index is found to be 3.95. The relative slope efficiencies of the four modes are summarized in Fig. 3(c). The modes at longer wavelengths have larger slope efficiency compared to the ones at shorter wavelengths. This was accompanied by a moderate emission droop for the modes at shorter wavelengths $(1188 \mathrm{~nm}$ and $1162 \mathrm{~nm})$ with higher injection power, as shown by the $\mathrm{L}-\mathrm{L}$ curves in Fig. 3(b). The higher slope efficiency of the longest wavelength performance suggests a higher capture efficiency in the QDs, as well as a slightly better confinement of the electron-hole pairs, leading to higher probability of radiative recombination.

At $300 \mathrm{~K}$, overlay of the emission spectra at the two extreme pump powers $(8.25 \mu \mathrm{W}$ and $495 \mu \mathrm{W})$ in Fig. 4(a) shows that the mode on the low-energy side wins in the mode competition and dominates the spectrum. The winning of the low-energy modes in the mode competition is related to the larger QD size, corresponding to the lower energy emission, and the superior capture efficiency. Mode spacing at this temperature slightly increases to $35 \mathrm{~nm}$ compared to the value of $29 \mathrm{~nm}$ at $10 \mathrm{~K}$, corresponding to a lower effective group index of 3.84 at $300 \mathrm{~K}$.

A rate equation analysis was carried out to fit the experimental $\mathrm{L}-\mathrm{L}$ curve to extract the spontaneous emission factor $\beta$.
We adopted a coupled rate equation model ${ }^{19,20}$ for the carrier density $\mathrm{N}$ and the photon density $\mathrm{P}$

$$
\begin{gathered}
\frac{d N}{d t}=\eta \frac{P_{i n}}{\hbar \omega V}-V_{g} g P-B N^{2}-C N^{3}, \\
\frac{d P}{d t}=V_{g} g P+\beta B N^{2}-\frac{P}{\tau_{p}},
\end{gathered}
$$

where $P_{i n}$ is the pump power, $\hbar \omega$ is the emitted photon energy, $V_{g}$ is the group velocity $\left(7.8 \times 10^{9} \mathrm{~cm} / \mathrm{s}\right)$, B is the bimolecular recombination coefficient $\left(2 \times 10^{-10} \mathrm{~cm}^{3} / \mathrm{s}\right)$, and $\mathrm{C}$ is the Auger coefficient $\left(8 \times 10^{-29} \mathrm{~cm}^{6} / \mathrm{s}\right)$. The photon lifetime $\tau_{p}(4.1 \mathrm{ps})$ is evaluated using the cold cavity quality factor (2950) obtained from sub-threshold measurements. Steady state solutions were found, assuming a gain function of $g=g_{0} \cdot \frac{N-N_{t}}{1+\varepsilon \cdot P}$, where $g_{0}$ is the differential gain $\left(3 \times 10^{-16}\right.$ $\left.\mathrm{cm}^{2}\right), \varepsilon$ is the gain compression factor $\left(1 \times 10^{-17} \mathrm{~cm}^{3}\right)$, and $N_{t}$ is the transparency carrier density $\left(1.23 \times 10^{18} \mathrm{~cm}^{-3}\right)$. Fig. 4(b) presents the experimental data and rate equation analytical fits using different values of $\beta$. The best fit to the experimental data reveals a high spontaneous emission coupling efficiency of $10 \%$. The nonlinear kink in the log-log L-L curve also occurs at the same pump power values as the kink in the linear scale L-L version (inset in Fig. 4(b)).

Figs. 5(a) and 5(b) present the temperature dependence of the lasing wavelength and spectral linewidth at twice the threshold pump power, respectively. The spectral positions of the mode redshift slightly at a rate of $\sim 0.04 \mathrm{~nm} / \mathrm{K}$ from $10 \mathrm{~K}$ to $80 \mathrm{~K}$, and from $220 \mathrm{~K}$ to $280 \mathrm{~K}$, which is ascribed to the temperature dependence of the cavity effective refractive index. The lasing line is at the same time governed by the temperature shrinkage of the active region bandgap, causing mode hopping towards longer wavelengths at $80 \mathrm{~K}$ 

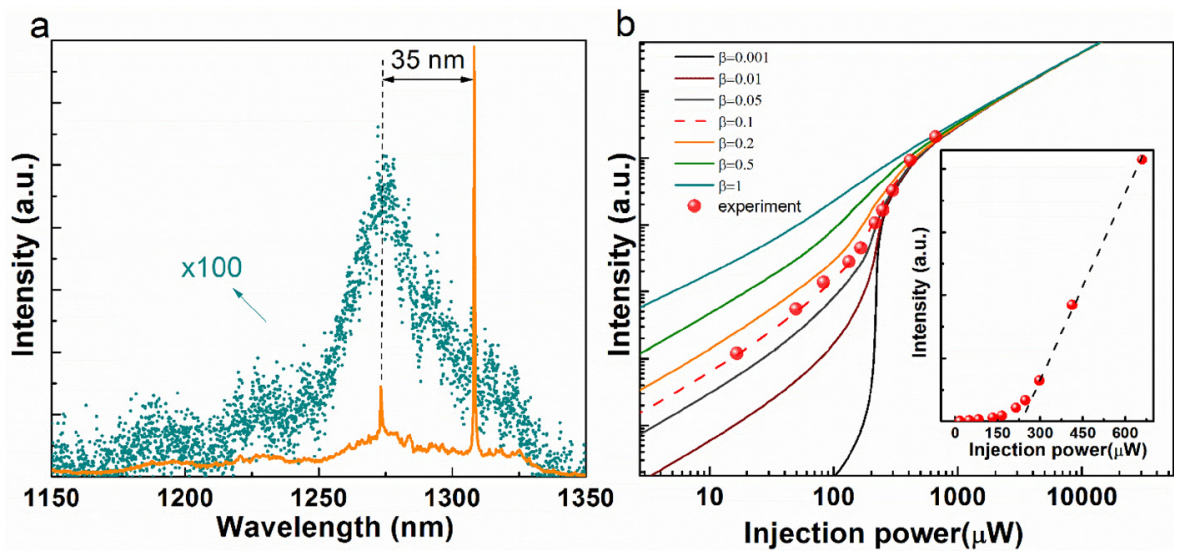

FIG. 4. (a) Laser emission spectra measured below (yellow, $8.25 \mu \mathrm{W}$ ) and above (light blue, $495 \mu \mathrm{W}$ ) threshold in $300 \mathrm{~K}$. Spectrum taken at $8.25 \mu \mathrm{W}$ was amplified 100 times to be visible; (b) L-L curve in the log-log scale in $300 \mathrm{~K}$. Rate equation model solutions for various values of $\beta$ are also presented. The best fit to the experimental data gives a spontaneous emission factor $\beta$ of 0.1 . Inset: $\mathrm{L}-\mathrm{L}$ curve in the linear scale, the dashed line represents a linear fit to the experimental data.

and $280 \mathrm{~K}$. The overall trend of the temperature dependent lasing line is governed by the confinement of the QDs, and the dependence of that confinement as a function of temperature, as well as the theoretical InAs band-gap shrinkage, as shown in Fig. 5(a). The theoretical InAs band-gap shrinkage is estimated via Varshni's formula, $\Delta E=A \cdot \frac{T^{2}}{T+B}$, assuming $\mathrm{A}=0.00042 \mathrm{eV} / \mathrm{K}^{2}$ and $\mathrm{B}=199 \mathrm{~K} .^{21}$ In Fig. 5(b), the linewidth slightly broadens to $0.4 \mathrm{~nm}$. We assume this to be related to the thermal redshift during the accumulation time of the spectrum measurement, which is a more prominent factor at higher temperatures. ${ }^{4}$ There is a certain fluctuation of linewidth with temperature, starting broad then reducing and then again increasing in irregular steps. This is probably due to varied amount of the spatial overlap of the QD gain spectral peak with the position of the cavity mode at different temperatures.

The threshold pump power as a function of temperature in Fig. 5(c) shows that the threshold increases by a factor of $\sim 2$ as the temperature increases from $10 \mathrm{~K}$ to $300 \mathrm{~K}$. This can be fit with an exponential function using $P_{t h} \alpha \exp \left(T / T_{0}\right),{ }^{19}$ and the characteristic temperature $\mathrm{T}_{0}$ was extracted to be around $105 \mathrm{~K}$. A slight decrease in the threshold from $10 \mathrm{~K}$ to $80 \mathrm{~K}$ is due to the suppressed multimode lasing at $80 \mathrm{~K}$. Otherwise, the experimental data follow the exponential fit, which increases at higher temperatures due to restrained carrier confinement in the QDs and enhanced non-radiative recombination. The relative slope efficiency decreases accordingly with increasing temperature, shown in Fig. 5(d). This is ascribed to the combined effects of free carrier absorption, intervalence band absorption, and suppressed constrains of defects. ${ }^{7}$

Figs. 6(a) and 6(b) plot the histograms of the threshold over a number of devices at $10 \mathrm{~K}$ and $300 \mathrm{~K}$, respectively. The average threshold is calculated to be $160 \mu \mathrm{W}$ at $10 \mathrm{~K}$ and $250 \mu \mathrm{W}$ at $300 \mathrm{~K}$, as illustrated in Fig. 6(c). The overall device thresholds only increase by a factor of $\sim 1.56$ when the temperature ramps from liquid helium temperature $(10 \mathrm{~K})$ up to room temperature $(300 \mathrm{~K})$, suggesting good thermal stability.

In conclusion, we performed systematic characterization of lasing behavior of the monolithically integrated microdisk lasers on a standard (001) silicon substrate. A high characteristic temperature $T_{o}$ of $105 \mathrm{~K}$ was extracted through the exponential fit of threshold power as a function of temperature from $10 \mathrm{~K}$ to $300 \mathrm{~K}$. At $300 \mathrm{~K}$, a high spontaneous emission coupling efficiency up to $10 \%$ is evidenced through rate equation analysis. The decent thermal stability and lasing characteristics suggest feasibility of manufacturing silicon chips with integrated micro-size lasers, promising to realize high-performance on chip optical links for telecommunication networks.
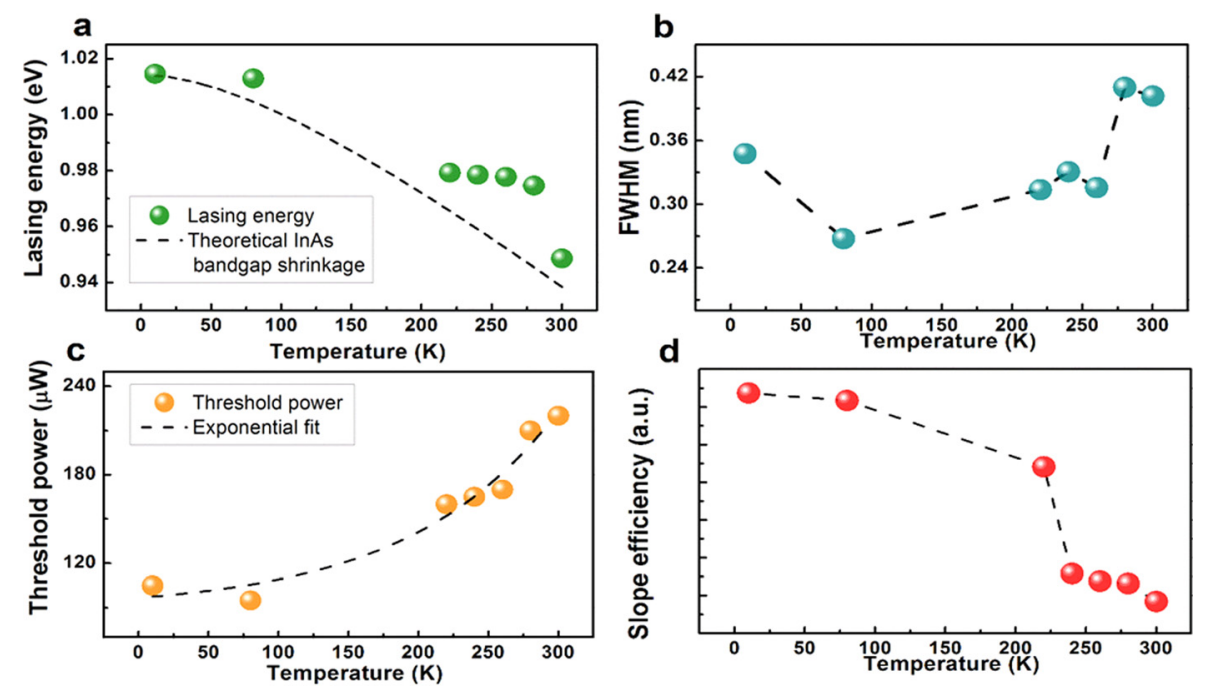

FIG. 5. (a)Temperature dependence of lasing wavelength at twice the threshold and theoretical InAs band-gap shrinkage; (b) temperature dependence of spectral linewidth at twice the threshold (c) temperature dependence of the threshold power, and the dashed line represents the exponential fit to the experimental data; and (d) temperature dependence of the relative slope efficiency. 

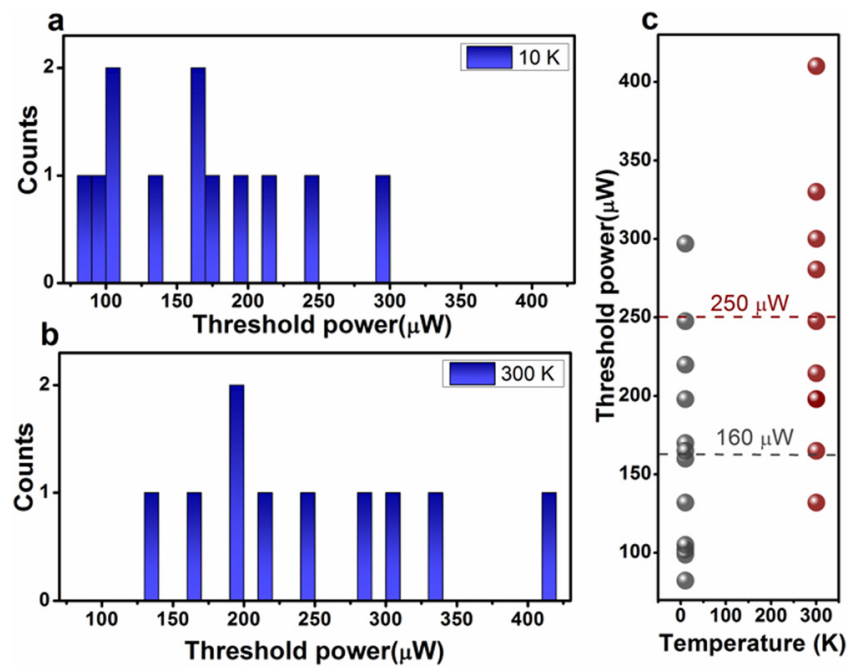

FIG. 6. Histograms of threshold over a number of devices at (a) $10 \mathrm{~K}$ and (b) $300 \mathrm{~K}$; (c) threshold power for a number of devices in $10 \mathrm{~K}$ (black spheres) and $300 \mathrm{~K}$ (red spheres). The dashed lines represent the average threshold.

This work was supported in part by Grant (Nos. 614813 and 16212115) from the Research Grants Council of Hong Kong and by a DARPA MTO EPHI Grant. The authors would like to thank AIM Photonics for providing the initial nano-patterned Si substrates, Wuhan National Laboratory for Optoelectronics (WNLO) for providing facilities to perform optical measurements, NFF and MCPF of HKUST for technical support. Helpful discussions with Z. Wang, C. Zeng, Y. Geng, and B. Shi are also acknowledged.

${ }^{1}$ M. T. Hill and Malte C. Gather, Nat. Photonics 8, 908 (2014).

${ }^{2}$ N. V. Kryzhanovskaya, A. E. Zhukov, M. V. Maximov, E. I. Moiseev, I. I. Shostak, A. M. Nadtochiy, Y. V. Kudashova, A. A. Lipovskii, M. M. Kulagina, and S. I. Troshkov, J. Sel. Top. Quantum Electron. 21, 709 (2015).
${ }^{3}$ N. V. Kryzhanovskaya, M. V. Maximov, A. E. Zhukov, A. M. Nadtochiy, E. I. Moiseev, I. I. Shostak, M. M. Kulagina, K. A. Vashanova, Y. M. Zadiranov, S. I. Troshkov, V. V. Nevedomsky, S. A. Ruvimov, A. A. Lipovskii, N. A. Kalyuzhnyy, and S. A. Mintairov, J. Lightwave Technol. 33, 171 (2015).

${ }^{4}$ T. Ide, T. Baba, J. Tatebayashi, S. Iwamoto, T. Nakaoka, and Y. Arakawa, Opt. Express 13, 1615 (2005).

${ }^{5}$ T. Yang, A. Mock, J. D. O’Brien, S. Lipson, and D. G. Deppe, Opt. Express 15, 7281 (2007).

${ }^{6}$ Y. Wan, Q. Li, A. Y. Liu, A. C. Gossard, J. E. Bowers, E. Hu, and K. M. Lau, Opt. Lett. 41, 1664 (2016).

${ }^{7}$ T. Ide, T. Baba, J. Tatebayashi, S. Iwamoto, T. Nakaoka, and Y. Arakawa, Appl. Phys. Lett. 85, 1326 (2004).

${ }^{8}$ Q. Li, K. W. Ng, and K. M. Lau, Appl. Phys. Lett. 106, 072105 (2015).

${ }^{9}$ Y. Wan, Q. Li, A. Y. Liu, W. W. Chow, A. C. Gossard, J. E. Bowers, E. Hu, and K. M. Lau, Appl. Phys. Lett. 108, 221101 (2016).

${ }^{10}$ A. Y. Liu, C. Zhang, A. Snyder, D. Lubyshev, J. M. Fastenau, A. W. K. Liu, A. C. Gossard, and J. E. Bowers, J. Vac. Sci. Technol. B 32, 02 C108 (2014).

${ }^{11}$ Y. Wan, Q. Li, Y. Geng, B. Shi, and K. M. Lau, Appl. Phys. Lett. 107, 081106 (2015).

${ }^{12}$ A. Lee, Q. Jiang, M. Tang, A. Seeds, and H. Liu, Opt. Express 20, 22181 (2012).

${ }^{13}$ M. Tang, S. Chen, J. Wu, Q. Jiang, V. G. Dorogan, M. Benamara, Y. I. Mazur, G. J. Salamo, A. Seeds, and H. Liu, Opt. Express 22, 11528 (2014).

${ }^{14}$ A. Y. Liu, C. Zhang, J. Norman, A. Snyder, D. Lubyshev, J. M. Fastenau, A. W. K. Liu, A. C. Gossard, and J. E. Bowers, Appl. Phys. Lett. 104, 041104 (2014).

${ }^{15}$ A. Y. Liu, R. W. Herrick, O. Ueda, P. M. Petroff, A. C. Gossard, and J. E. Bowers, IEEE J. Sel. Top. Quantum Electron. 21, 1900708 (2015).

${ }^{16}$ A. Y. Liu, S. Srinivasan, J. Norman, A. C. Gossard, and J. E. Bowers, Photonics Res. 3, B1 (2015).

${ }^{17}$ S. Chen, W. Li, J. Wu, Q. Jiang, M. Tang, S. Shutts, S. N. Elliott, A. Sobiesierski, A. J. Seeds, I. Ross, P. M. Smowton, and H. Liu, Nat. Photonics 10, 307 (2016).

${ }^{18}$ A. C. Tamboli, E. D. Haberer, R. Sharma, K. H. Lee, S. Nakamura, and E. L. Hu, Nat. Photonics 1, 61-64 (2007).

${ }^{19}$ L. A. Coldren, S. W. Corzine, and M. L. Mashanovitch, Diode Lasers and Photonic Integrated Circuits (Wiley, 2012), Vol. 218.

${ }^{20}$ Z. Wang, B. Tian, M. Pantouvaki, W. Guo, P. Absil, J. V. Campenhout, C. Merckling, and D. V. Thourhout, Nat. Photonics 9, 837 (2015).

${ }^{21}$ R. Heitz, I. Mukhametzhanov, A. Madhukar, A. Hoffmann, and D. Bimberg, J. Electron. Mater. 28, 520 (1999). 